Descargo de responsabilidad: las opiniones expresadas en los manuscritos son responsabilidad exclusiva de los autores. No necesariamente reflejan las opiniones de la editorial ni la de sus miembros.

1 CENIRO

\title{
Integración de las diferentes culturas en la legislación en el territorio guatemalteco
}

\section{Integration of the Different Cultures in the legislation in the Guatemalan territory}

Recibido: $04 / 11 / 2021$

Publicado: 20/01/2022

\author{
Karenn Janeth Medina Rivas \\ Maestría en Derecho Penal \\ Universidad de San Carlos de Guatemala \\ karenn@postgradocunzac.edu.gt \\ https://orcid.org/0000-0002-3888-1312
}

\section{Referencia}

Medina Rivas, K. J. (2022). Integración de las diferentes culturas en la legislación en el territorio guatemalteco. Revista Académica Sociedad Del Conocimiento Cunzac, 2(1), 65-70. DOI: https://doi.org/10.46780/sociedadcunzac.v2i1.14

\section{Resumen}

OBJETIVO: establecer la problemática relativa a los delitos culturales motivados debido a una diversidad cultural originada en relación a los procesos multiculturales vinculados a los pueblos indígenas de América Latina. MÉTODO: por medio de la metodología de investigación bibliográfica se llegó a establecer que la razón el derecho penal se construyó sobre los valores de una sociedad, el multiculturalismo necesariamente ha atravesado los límites culturales y sociales de esta. RESULTADOS: se pudo establecer con base a los hallazgos encontrados que como es aplicable el derecho penal a una persona que no comprende, por el elaborado de sus prácticas originarias, que una acción puede constituir un realizado penalmente sancionado. Es decir, algo que en Guatemala no se observa, debido a que la regla jurídica instituye que no se puede alegar ignorancia frente a la observancia de la ley, por lo cual no podría pensarse que una acción penalmente regulada como delito un juez logre considerarla como una acción clásica no antijurídica por el elaborado de que en su sociedad no se considera como delito. CONCLUSIÓN: Guatemala un país multiétnico es importante que en la legislación penal se integren las diferentes culturas de los diversos grupos existentes en el territorio nacional, no sufran o se sientan discriminados y que se les respeten las costumbres propias de su identidad cultural.

\section{Palabras clave}

modelo, regulación, integración, cultura, reconocimiento

\section{Abstract}

OBJECTIVE: to establish the problem related to cultural crimes motivated by cultural diversity originated in relation to multicultural processes linked to indigenous peoples of Latin America. METHOD: through the bibliographic research methodology it was established that the reason criminal law was built on the values of a society, multiculturalism has necessarily crossed the cultural and social limits of this. RESULTS: it was possible to establish based on the findings found that how criminal law is applicable to a person who does not understand, due to 
the elaboration of their original practices, that an action can constitute a criminally sanctioned act. That is to say, something that is not observed in Guatemala, because the legal rule establishes that ignorance can not be alleged regarding the observance of the law, for which it could not be thought that a criminal action regulated as a crime by a judge could consider it as a crime. Classical non-illegal action because in their society it is not considered a crime. CONCLUSION: in Guatemala, a multi-ethnic country, it is important that the criminal legislation integrates the different cultures of the various groups existing in the national territory, that they do not suffer or feel discriminated against, and that the customs of their cultural identity are respected.

\section{Keywords}

model, regulation, integration, culture, recognition

\section{Introducción}

En la aplicación de un ordenamiento jurídico que pertenece a una cultura determinada se hace evidente el considerar los componentes como la diversidad cultural, el pluralismo jurídico, la ciudadanía cultural, los derechos culturales, los derechos de los pueblos originarios y el etnocentrismo. En el caso peruano se plantea la situación de forma intensificada porque existen diferencias culturales incluyendo las de orden jurídico, de los distintos grupos de la población donde son más marcadas las injusticias y grandes desigualdades. Para ejemplificar en el Artículo 15 del código Penal peruano constantemente sufre problemas de interpretación por la ineficiente comprensión de ese texto, en el que su objetivo ha sido presentar doctrina y jurisprudencia peruana aplicando criterios y disposiciones referentes a como se regulan los efectos que se presentan en los conflictos culturales, teniendo en cuenta que las pautas culturales del infractor son distintas a las que están establecidas en la ley penal oficial (Ballón, 2002 p. 74).

Interpretando el artículo antes citado el que por su cultura o prácticas comete un elaborado punible sin poder entender el carácter delictuoso de su acto o determinarse según dicha comprensión, va a ser eximido de responsabilidad. Según Basilico quien sigue a Hurtado y a Kalinsky, opina que el infractor se desarrolla en una "cultura distinta a la nuestra" y que "ha internalizado desde niño las pautas de conducta de esa cultura". (Basilico, 2017 p. 85).

Es decir que lo que para la legislación aparece como una infracción a la ley penal para el considerado infractor es una conducta que forma parte de su cultura. Para definir el error se debería decir que consiste en que una persona desacierta en juzgar su comportamiento como lícito porque no ha asimilado o no es de su conocimiento o entendimiento la norma que lo prohíbe, o porque aprecia que su norma cultural es predominante sobre la norma prohibitiva, entendiéndose que sabe que existe la norma pero que ésta es de una cultura que le es ajena. Citando a Peña, hace referencia que "se ha regulado el error de prohibición y que el Perú es étnica y culturalmente plural, estimó necesario que en el nuevo código se resaltase normativamente el respeto de los valores culturales distintos "en el sentido que no se criminalice conductas socialmente aceptadas en los grupos culturales" (Peña, 1994 p. 436-444). Haciendo referencia a lo considerado por los anteriores autores resulta necesario crear un modelo de legislación en donde no se criminalice al infractor por conductas que han sido aceptadas socialmente en las diferentes culturas, es decir que se respeten los valores culturales distintos. 
Existe en la actualidad inconvenientes de interpretación legal, ineficiente e incomprensible, en el cual su objetivo fue exponer ideología y jurisprudencia, y, cómo se utilizan criterios y posiciones referentes a cómo regular los efectos que se muestran en los conflictos culturales, teniendo presente que las pautas culturales del transgresor son diversas a las que permanecen establecidas en la ley penal oficial.

De acuerdo a la problemática sobre la deficiente e incomprensible interpretación legal existen muchos puntos de vista con la interpretación de principios, normas, doctrinas, y jurisprudencia con base a las diferentes culturas que predominan que se dificulta la estandarización de las normativas, como consecuencia de la conducta humana. Por tal razón es necesaria la integración de las diferentes culturas en la legislación en el territorio guatemalteco con el propósito de que no sean juzgados por actuaciones que para ellos son parte de su cultura.

\section{Contenido:}

\section{Valores culturales de los diferentes grupos sociales}

El presente artículo científico tiene que ver con el 15 del Código Penal peruano y del error de comprensión culturalmente condicionado, hay varias teorías que tienen la posibilidad de tener en cuenta que vulnera los derechos primordiales a el equilibrio del individuo humano y el reconocimiento de la variedad cultural consagrados en los incisos 2 y 19 del artículo $2^{\circ}$ de la Constitución.

No obstante, autores como Zaffaroni y Peña, indican que la norma jurídica no criminaliza las conductas de los nativos que en determinados casos pueden contravenir el ordenamiento jurídico ordinario del país. Existen dos clases de persona a las que se puede aplicar la norma, las primeras de las cuales es aquella persona que conoce la norma prohibitiva de la conducta, pero que no la considera como suya por razón de su cultura y la persona que por motivos de aislamiento de la sociedad convencional no conoce que la norma es prohibitiva de ciertas conductas. (Zaffaroni, et al., 2000 p. 411).

En el primero de los casos se trata de la internalización de la norma, la persona no considera que deba aceptar la normas como suya porque en su cultura no es una forma normal en la que se actúe, pero se crea una controversia, si la acción prohibida es cometida no dentro de su comunidad, sino en el territorio en donde se aplica la ley ordinaria, ¿cómo se debe juzgar esta conducta?

En el segundo de los casos no habría ningún problema con respecto a detectar la comisión de un hecho considerado como delictivo ya que el lugar de la comisión del mismo sería la misma comunidad étnica, donde la conducta se considera legal y no podría ser objeto de denuncia. El error de comprensión culturalmente condicionado debería entenderse como un error propiamente hablado, que imposibilita la comprensión de la antijuridicidad del comportamiento, originado por el condicionamiento cultural del sujeto. Al indicar un error que, por su carácter invencible, excluye la responsabilidad y toda sanción penal, debido a que, siguiendo el marco predeterminado de los recursos del delito, los cuales son la tipicidad, antijuricidad y responsabilidad, de no cumplirse uno de ellos no podría configurarse un delito. 
Sin embargo, esto crea la posibilidad de que se crea que la persona por éste motivo es inimputable, lo cual en cierto momento se ha considerado como una afrenta a la diversidad cultural (Zaffaroni, 2000, p. 411). Dejó de lado una vez que una persona, por su cultura o costumbre, no puede entender el carácter ilícito del acto, entonces, se le exime de responsabilidad o se le atenúa si dicha capacidad de comprensión está atenuada.

La terminología del artículo 15 del Código Penal peruano es muy criticada por los motivos anteriormente expuestos; sin embargo, en comparación con la legislación guatemalteca que es mucho más inclusiva y permite que sea aplicado de mejor forma el ordenamiento jurídico consuetudinario, ya que en la actualidad la norma jurídica regula que nadie puede alegar ignorancia ante la observancia de la ley, establecido en la Ley del Organismo Judicial de Guatemala en su artículo 3, (Organismo Legislativo, 1989), por lo que tener una norma que establezca que el que por su cultura o costumbre comete un acto punible sin poder concebir el carácter delictuoso de su acto o comprobar según dicha comprensión, va a ser eximido de responsabilidad. Una vez que igualmente razón, dicha probabilidad se encuentra disminuida, se atenúa la pena, evitaría la utilización de peritajes culturales en los procesos en los cuales se diligencian en casos por ejercerse una acción penalmente reprochable empero permitida en la cultura a la cual pertenece.

Es importante el análisis de este artículo para poder establecer como es aplicable el derecho penal a una persona que no comprende, por el hecho de sus costumbres nativas, que una acción puede constituir un hecho penalmente sancionado. Esta forma de legislar permite en cierto momento que una persona que cometió un hecho que la ley regula como delictivo pueda considerarse como no delito dentro de su comunidad indígena y que no sea sancionado o bien la pena sea atenuada. Esto es algo que en Guatemala no se observa, ya que la norma jurídica establece que no se puede alegar ignorancia ante la observancia de la ley, por lo que no podría pensarse que una acción penalmente regulada como delito un juez pueda considerarla como una acción típica no antijurídica por el hecho de que en su comunidad no es considerada como delito.

En síntesis, el objetivo de este artículo es incluir un modelo de legislación en donde se resalten los valores culturales de los diferentes grupos sociales, y se les respeten las normas de conducta propias de dicha cultura debido a que en Guatemala no se puede alegar ignorancia ante la observancia de la ley.

\section{Conclusión}

Según la problemática que se presenta en países desarrollados, sobre las diferentes teorías en relación a las culturas o costumbres, y sobre los hechos punibles; sin poder comprender el carácter delictuoso, y sobre si debe o no debe ser eximido de su responsabilidad; llegándose a la conclusión de que debe existir en Guatemala un modelo de integración en la legislación que permita el reconocimiento de las diferentes culturas en el territorio nacional.

Por ser Guatemala un país multiétnico es importante que en la legislación penal se integren las diferentes culturas de los diversos grupos existentes en el territorio nacional, no sufran o se sientan discriminados y que se les respeten las costumbres propias de su identidad cultural. 


\section{Referencias}

Ballón, F. (2002) Introducción al derecho de los pueblos indígenas, Lima Perú. https://es.scribd. com/document/315266587/Ballon-Aguirre-Francisco-Introduccion-al-derecho-de-los-pueblos-indigenas-pdf

Basilico, R. (2017) "Error de prohibición culturalmente motivado, Diversidad cultural y administración de justicia", Cathedra Jurídica, Buenos Aires. https://1library.co/document/ yjor936z-la-relevancia-de-la-motivacion-cultural-codigo-penal.html

Organismo Legislativo. (1989). Decreto 2-89. Ley de Organismo Judicial. 10 de enero de 1989. https://leyes.infile.com/visualizador_demo/index.php?id=21109\#principio

Peña, R. (1994) Derecho penal. Estudio programático, Lima, Grijley. https://derecho.usmp.edu. pe/instituto/novedades/ibro-teoria-del-delito-oscar-pena.pdf

Zaffaroni, E. R., Alagia, A., \& Slokar, A. (2000). Derecho penal: parte general (Vol. 2). Buenos Aires: Ediar. https://www.zonalegal.net/uploads/documento/Zaffaroni-Manual\%20de\%20 Derecho\%20Penal\%20Parte\%20General\%20(Ed\%202\%202006)\%20(1).pdf

\section{Sobre la autora}

Maestra de Educación Primaria Urbana, labora para el Magisterio Nacional de Guatemala desde el año de 1993 hasta la fecha. Profesora de Enseñanza Media en Pedagogía y Ciencias de la Educación, egresada de la Universidad de San Carlos de Guatemala. (2000) Abogada y Notaria, egresada de la Universidad Mariano Gálvez, con 3 años de ejercicio Profesional. Colegiado No. 32072. Actualmente es estudiante del tercer semestre de Maestría en Derecho Procesal Penal en el Centro Universitario de Zacapa (CUNZAC) de la Universidad San Carlos de Guatemala.

\section{Financiamiento de la investigación}

Con recursos propios de los investigadores

\section{Declaración de intereses}

Declaran no tener ningún conflicto de intereses, que puedan haber influido en los resultados obtenidos o las interpretaciones propuestas.

\section{Declaración de consentimiento informado}

El estudio se realizó respetando el Código de ética y buenas prácticas editoriales de publicación. 


\section{Derechos de uso}

Copyright@ 2022 por Karenn Janeth Medina Rivas. Este texto está protegido por una licencia Creative Commons 4.0

Este texto está protegido por la Licencia Creative Commons Atribución 4.0 Internacional.

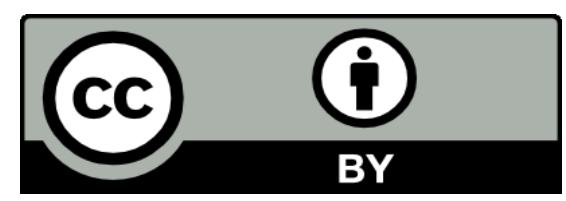

Este texto está protegido por una licencia Creative Commons 4.0.

Es libre para compartir, copiar y redistribuir el material en cualquier medio o formato y adaptar el documento, remezclar, transformar y crear a partir del material para cualquier propósito, incluso comercialmente, siempre que cumpla la condición de atribución: debe reconocer el crédito de una obra de manera adecuada, proporcionar un enlace a la licencia, e indicar si se han realizado cambios. Puede hacerlo en cualquier forma razonable, pero no de forma tal que sugiera que tiene el apoyo del licenciante o lo recibe por el uso que hace. 